\title{
PRIMEROS ESTUDIOS CARIOTÍPICOS EN MIMOSA FARINOSA Y M. VELLOZIANA (LEGUMINOSAE, MIMOSOIDEAE)
}

\author{
MATÍAS MORALES ${ }^{1,2,3,5}$, ARTURO F. WULFF ${ }^{3,4}$, RENÉE H. FORTUNATO ${ }^{1,2,3}$ \& LIDIA POGGIO RI, $^{3,4}$
}

\begin{abstract}
Summary: Morales, M., A. F. Wulff, R. H. Fortunato \& L. Poggio. 2015. First karyotype studies in M. farinosa and M. velloziana (Leguminosae, Mimosoideae). Bonplandia 24(1): 57-62.

The first karyotype studies in Mimosa farinosa and $M$. velloziana are presented. The chromosome number of $M$. farinosa, $2 n=2 x=26$ (Mimosa section Batocaulon series Farinosae) is mentioned for the first time, while the chromosome number of $M$. velloziana var. velloziana, $2 n$ $=4 \mathrm{x}=52$ (Mimosa section Mimosa series Mimosa subseries Mimosa) is confirmed. The formula and parameters of karyotype (chromosome length and A1 and A2 asymmetry indexes) were analyzed. The karyotype formula of $M$. farinosa $(16 \mathrm{~m}+10 \mathrm{sm})$ and $M$. velloziana var. velloziana $(48 \mathrm{~m}+4 \mathrm{sm})$ were calculated. Mimosa farinosa has a more asymmetric karyotype and larger chromosome size than M. velloziana var. velloziana. A1 index, total chromosome length, and chromosome length per haploid genome showed significant differences between the two taxa. These results agree with differences previously found among other members of series Farinosae and subseries Mimosa, suggesting that certain karyotype parameters could have cytotaxonomic interest in some groups of species of this genus.
\end{abstract}

Key words: Chromosome asymmetry, cytogenetics, polyploidy, series Farinosae, subseries Mimosa.

Resumen: Morales, M., A. F. Wulff, R. H. Fortunato \& L. Poggio. 2015. Primeros estudios cariotípicos en Mimosa farinosa y M. velloziana (Leguminosae, Mimosoideae). Bonplandia 24(1): 57-62.

Se presentan los primeros estudios de cariotipo en Mimosa farinosa y M. velloziana. Se cita por primera vez el número cromosómico de $M$. farinosa, $2 n=2 x=26$ (Mimosa sección Batocaulon serie Farinosae) y se confirma el número cromosómico de $M$. velloziana var. velloziana: $2 \mathrm{n}=$ $4 \mathrm{x}=52$ (Mimosa sección Mimosa serie Mimosa subserie Mimosa). Se analizó la fórmula y los parámetros del cariotipo (longitud cromosómica e índices de asimetría $\mathrm{A}_{1}$ y $\mathrm{A}_{2}$ ). Se determinaron las fórmulas cariotípicas de $M$. farinosa $(16 \mathrm{~m}+10 \mathrm{sm})$ y de $M$. velloziana var. velloziana $(48 \mathrm{~m}+4 \mathrm{sm})$. M. farinosa posee un cariotipo más asimétrico y mayor tamaño cromosómico que M. velloziana var. velloziana. El índice $A_{1}$, el largo cromosómico total y el largo cromosómico por genoma haploide exhibieron diferencias significativas entre ambos taxones. Estos resultados concuerdan con diferencias halladas previamente entre otros representantes de la serie Farinosae y la serie Mimosa subserie Mimosa, lo que sugiere que algunos parámetros evaluados tienen interés citotaxonómico en grupos de especies de este género.

Palabras clave: Asimetría cromosómica, citogenética, poliploidía, serie Farinosae, subserie Mimosa.

${ }^{1}$ Instituto de Recursos Biológicos, CIRN-CNIA, INTA. N. Repetto \& Los Reseros s.n. (1686) Hurlingham. Pcia. De Buenos Aires. República Argentina.

${ }^{2}$ Consejo Nacional de Investigaciones Científicas y Técnicas (CONICET). República Argentina.

${ }^{3}$ Facultad de Agronomía y Ciencias Agroalimentarias, Universidad de Morón. Cabildo 134 (B1708JPD) Morón. Pcia. de Buenos Aires. República Argentina.

${ }^{4}$ Departamento de Ecología, Genética y Evolución, Facultad de Ciencias Exactas y Naturales, Universidad de Buenos Aires, Intendente Güiraldes y Costanera Norte (CI42EHA), Ciudad Autónoma de Buenos Aires. República Argentina.

${ }^{5}$ Autor corresponsal, e-mail: mmorales0007@gmail.com 


\section{Introducción}

El género Mimosa L. es uno de los más diversos entre los de la familia Leguminosae y la subfamilia Mimosoideae, y comprende actualmente más de 530 especies (Simon et al., 2011). Es un género mayormente de distribución pantropical y pan-subtropical, altamente diversificado en Sudamérica (Barneby, 1991). Mimosa fue dividido en cinco secciones: Mimadenia Barneby, Habbasia DC., Batocaulon DC., Calothamnos Barneby y Mimosa.

El número cromosómico básico postulado para este género es $\mathrm{x}=13$ (Isely, 1971; Goldblatt, 1981), y se han propuesto diferentes niveles de ploidía: $2 \mathrm{n}=2 \mathrm{x}=26,2 \mathrm{n}=3 \mathrm{x}=$ $39,2 \mathrm{n}=4 \mathrm{x}=52,2 \mathrm{n}=6 \mathrm{x}=78$ y $2 \mathrm{n}=8 \mathrm{x}$ $=104$ (Seijo, 1993, 1999, 2000; Seijo \& Fernández, 2001; Dahmer et al., 2011; Olkoski \& Schifino-Wittmann, 2011; Morales et al., 2010, 2011, 2012, 2014a, b). La mayoría de las especies son diploides y tetraploides; en menor medida, se han hallado triploides, hexaploides y octoploides, especialmente en el S de Sudamérica (Seijo, 1993, 1999, 2000; Seijo \& Fernández, 2001; Dahmer et al., 2011; Morales et al., 2014a, b). La poliploidía tiene una alta incidencia en este género, y aparece aproximadamente en un 30-35\% de las especies estudiadas (Seijo, 2000; Dahmer et al., 2011; Morales et al., 2014a). Asimismo, parece darse con mayor frecuencia en los grupos diversificados en el área meridional de distribución y en las más elevadas latitudes (Seijo, 1999, 2000; Seijo \& Fernández, 2001; Dahmer et al., 2011; Morales et al., 2014a).

Los estudios cariotípicos en Mimosa se han realizado en un número relativamente reducido de especies. Morales et al. (2011) estudiaron el cariotipo de siete especies del $\mathrm{S}$ de Sudamérica, de las secciones Batocaulon y Mimosa. En estos estudios se observó que existen diferencias en fórmula cariotípica y tamaño cromosómico entre especies de ambas secciones, si bien en líneas generales, el cariotipo es simétrico y el tamaño cromosómico pequeño. Recientemente, Marçal de Souza et al. (2012) estudiaron el cariotipo de $M$. caesalpiniifolia Benth., especie en la cual predominan los cromosomas metacéntricos y submetacéntricos y donde se ha informado la presencia de cromosomas B.

La escasez de estudios cariotípicos en este género se debe mayormente a las dificultades técnicas existentes para visualizar el centrómero y lograr una buena dispersión de células en metafase con las técnicas tradicionales de tinción, así como al tamaño reducido de los cromosomas (Seijo, 2000).

Con el objetivo de incrementar los estudios cariotípicos en Mimosa, y en particular en determinados grupos de especies donde estos análisis se iniciaron previamente, se presenta el número cromosómico, fórmula y parámetros del cariotipo de $M$. farinosa Griseb. y $M$. velloziana Mart. var. velloziana. Los datos son discutidos en el contexto de los últimos antecedentes citogenéticos en este género.

\section{Materiales y Métodos}

Los especímenes de herbario de referencia, así como los materiales para estudios citogenéticos, fueron obtenidos a partir de individuos cultivados en el Jardín Botánico “Arturo Ragonese”, en el caso de $M$. farinosa, y de individuos al estado silvestre recolectados en el $\mathrm{N}$ de Argentina, en el caso de $M$. velloziana.

Especímenes de referencia: Mimosa farinosa: ARGENTINA. Buenos Aires: Pdo. Hurlingham, cultivado en INTA-Castelar, procedencia $\mathrm{N}$ de Córdoba, 14-II-2006, Morales 194 (BAB).

M. velloziana var. velloziana: ARGENTINA. Salta: Dpto. Orán, camino a Isla de Cañas, Finca San Ignacio, 30-IX-2005, Morales et al. 125 (BAB); entre Agua Blanca y Angosto del Pescado, RP19, 19-IX-1991, Guaglianone 2726 (SI, CTES 186422).

Semillas correspondientes al material de referencia de ambas especies (Tabla 1) germinaron en cajas de Petri a $25^{\circ} \mathrm{C}$. Cuando las raicillas alcanzaron $1-2 \mathrm{~cm}$ de largo, se colocaron en 8-hidroquixinoleína 0,002 M durante 4-6 h, y se fijaron en solución 3:1 etanol absoluto:ácido acético glacial. Pasados 24-48 h se trasvasaron a etanol $75 \%$ y se conservaron a $5^{\circ} \mathrm{C}$ hasta el momento de su uso.

Para la confección de los preparados mitóticos, el material conservado en etanol se 
trasvasó a una solución buffer $0,01 \mathrm{M}$ ácido cítrico-citrato de sodio a $\mathrm{PH}=4,6$. Luego se transfirió a una solución enzimática constituida por $2 \mathrm{ml}$ celulasa 2\% (Ozonuka R-10, Merck $\mathrm{KGaA}$, Damstadt, Alemania) y pectinasa líquida $20 \%$, durante $90-120 \mathrm{~min}$ a $37^{\circ} \mathrm{C}$. El material se lavó posteriormente con la solución buffer descripta. Finalmente, se realizó una tinción convencional con hematoxilina acética/ácido acético $45 \%$ y se aplicó la técnica de "squash". Se seleccionaron y fotografiaron al menos 5 células en prometafase/metafase por cada preparado, con un fotomicroscopio Leica LM2500.

En las células seleccionadas se midió el Largo Cromosómico Total (LCT) y se calculó el Largo Cromosómico por Genoma Haploide (LCGH). La longitud total de cada cromosoma, así como la longitud de los brazos se midieron con el programa Micromeasure ${ }^{\circledR}$ (Reeves, 2001). Se confeccionó el cariotipo de ambos taxones, determinando su fórmula cariotípica. Se utilizó la nomenclatura de Levan et al. (1964) y los cromosomas fueron clasificados de acuerdo con el Índice Centromérico (Ic). Se calcularon los índices de asimetría $\mathrm{A}_{1}$ y $\mathrm{A}_{2}$ (Romero-Zarco, 1986).

Con el objeto de detectar diferencias significativas entre los taxones en los parámetros calculados (LCT, LCGH, $\mathrm{A}_{1}, \mathrm{~A}_{2}$ ), se realizó un test $t$ de Student para dos muestras independientes, utilizando el programa Infostat (Di Rienzo et al., 2009) con un nivel de significación del 5\%.

\section{Resultados}

El número cromosómico determinado para $M$. farinosa es $2 \mathrm{x}=2 \mathrm{n}=26$, mientras que el número cromosómico determinado para $M$. velloziana var. velloziana es $2 \mathrm{n}=4 \mathrm{x}=52$ (Fig. 1). La fórmula cariotípica observada en $M$. farinosa fue $16 \mathrm{~m}+10 \mathrm{sm}$, mientras que la fórmula cariotípica observada en $M$. velloziana var. velloziana fue $48 \mathrm{~m}+4 \mathrm{sm}$. Este último taxón es tetraploide, y si bien no se conoce su origen, fue posible agrupar los cromosomas de a pares debido a su número y morfología (Fig. 2; Tabla 1).

En cuanto a parámetros de tamaño cromosómico, $M$. farinosa posee cromosomas de mayor tamaño que $M$. velloziana var. velloziana, ya que existen diferencias significativas entre ambos taxones tanto en LCT como en LCGH; éste último parámetro posee mayor valor comparativo entre diploides y poliploides debido a que elimina la incidencia de los diferentes niveles de ploidía. En cuanto a los índices de asimetría, el índice $\mathrm{A}_{1}$ mostró diferencias significativas entre ambos taxones, mientras que no se observaron diferencias al analizar el índice $\mathrm{A}_{2}$ (Tabla 1).
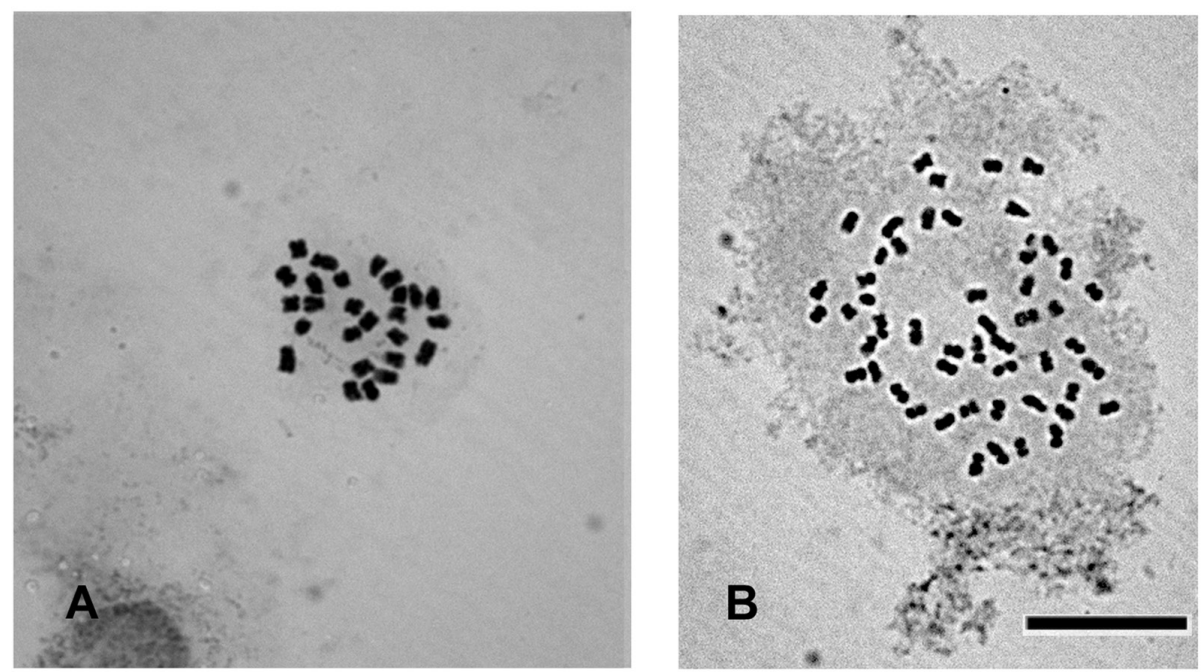

Fig. 1. Células somáticas de Mimosa en metafase. A: . farinosa, $2 \mathrm{n}=2 \mathrm{x}=26$. B: M. velloziana var. velloziana, $2 \mathrm{n}=$ $4 \mathrm{x}=52$. La barra indica $10 \mu \mathrm{m}$. 


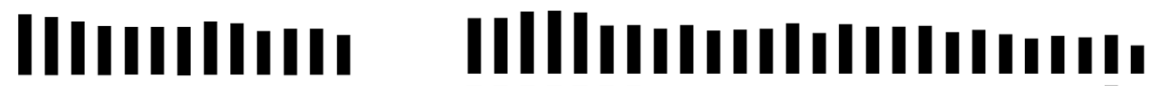

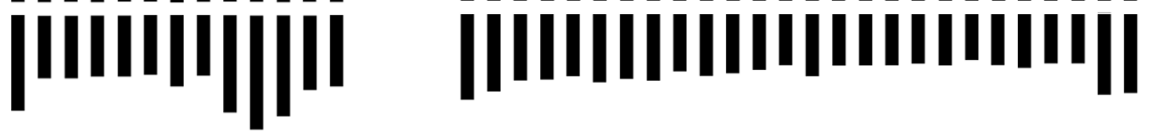 \\ m sm \\ A

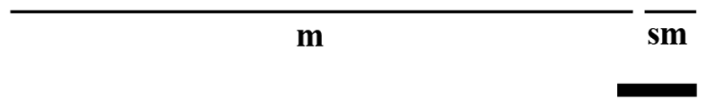 \\ B}

Fig. 2. Cariogramas de especies de Mimosa. A: M. farinosa, $2 \mathrm{n}=2 \mathrm{x}=26$. B: M. velloziana var. velloziana, $2 \mathrm{n}=4 \mathrm{x}$ $=52 ; \mathrm{m}=$ pares de cromosomas metacéntricos; $\mathrm{sm}=$ pares de cromosomas submetacéntricos. La barra indica $1 \mu \mathrm{m}$.

Tabla 1. Número cromosómico, fórmula y parámetros del cariotipo en Mimosa farinosa y M. velloziana var. velloziana.

\begin{tabular}{|ccccc|}
\hline & M. farinosa & $\begin{array}{c}\text { M. velloziana var. } \\
\text { velloziana }\end{array}$ & $\begin{array}{c}\text { Estadístico } \boldsymbol{t} \\
\text { obtenido }\end{array}$ & valor $\boldsymbol{p}$ \\
\hline Número cromosómico & $2 \mathrm{n}=2 \mathrm{x}=26$ & $2 \mathrm{n}=4 \mathrm{x}=52$ & - & - \\
LCT $(\mu \mathrm{m})$ & $40,56 \pm 7,68$ & $58,14 \pm 5,00$ & $-3,65$ & 0,0045 \\
LCGH $(\mu \mathrm{m})$ & $20,28 \pm 3,74$ & $14,54 \pm 1,25$ & 2,48 & 0,0328 \\
Fórmula cariotípica & $16 \mathrm{~m}+10 \mathrm{sm}$ & $48 \mathrm{~m}+4 \mathrm{sm}$ & - & - \\
$\mathrm{A}_{1}$ & $0,39 \pm 0,01$ & $0,20 \pm 0,02$ & 13,54 & 0,0009 \\
$\mathrm{~A}_{2}$ & $0,20 \pm 0,04$ & $0,17 \pm 0,02$ & 1,15 & 0,2784 \\
\hline
\end{tabular}

\section{Discusión}

El número cromosómico $2 \mathrm{n}=2 \mathrm{x}=26 \mathrm{de}$ $M$. farinosa es inédito, mientras el número cromosómico $2 \mathrm{n}=4 \mathrm{x}=52$ de $M$. velloziana var. velloziana es una confirmación de recuentos anteriores (Seijo, 1999; Dahmer et al., 2011). Al igual que la mayor parte de los antecedentes mencionados en la introducción, los números cromosómicos encontrados concuerdan con que $\mathrm{x}=13$ sería el número cromosómico básico del género Mimosa.

Mimosa farinosa es una especie endémica del Chaco Sudamericano y áreas adyacentes (Barneby, 1991). Pertenece a la sección Batocaulon serie Farinosae Barneby, un grupo de árboles y arbustos xerófitos de las áreas semiáridas templadas y subtropicales del $\mathrm{S}$ de Sudamérica. Este grupo comprende cuatro especies (Barneby, 1991); en tres de ellas, $M$. detinens Benth., M. ostenii Burkart y $M$. farinosa, se conoce su número cromosómico $(2 \mathrm{n}=2 \mathrm{x}=26)$. El cariotipo de $M$. farinosa exhibe gran similitud con el de las otras especies de Farinosae previamente estudiadas, M. detinens y M. ostenii (Morales et al., 2011). Las tres poseen los cariotipos más asimétricos entre las especies de Mimosa estudiadas hasta el momento, así como los cromosomas de mayor tamaño. En la serie Farinosae, la fórmula cariotípica incluye sólo 7-9 pares de cromosomas metacéntricos; el resto son submetacéntricos; la LCT es mayor a $40 \mu \mathrm{m}$ y los índices de asimetría, son relativamente altos en comparación con el resto de las especies estudiadas de la sección Mimosa (Morales et al., 2011; Tabla 1). Los altos valores en parámetros de tamaño cromosómico de las especies de la serie Farinosae podrían corresponder a un mayor tamaño del genoma en relación al resto de las especies estudiadas de Mimosa.

La asimetría intracromosómica en $M$. farinosa, M. detinens y M. ostenii (Morales et al., 2011) exhibe mayores diferencias con otras especies de Mimosa que la intercromosómica. Los valores del índice $\mathrm{A}_{1}$ en estas tres especies 
oscilan en el rango 0,34-0,39, mientras que en casi todas las demás especies estudiadas del género son menores a 0,3 (Morales et al., 2011). En cambio, el índice de asimetría $\mathrm{A}_{2}$ en la serie Farinosae oscila en el rango habitual de las especies de Mimosa estudiadas hasta el momento: 0,14-0,27 (Morales et al., 2011, 2014a, b). Como otra característica importante que se repite en las restantes especies del género estudiadas, no se han encontrado hasta el momento cariotipos bimodales, lo cual explica que los valores de $\mathrm{A}_{2}$ son muy pequeños.

Mimosa velloziana var. velloziana pertenece a la sección Mimosa serie Mimosa subserie Mimosa, y es un taxón ampliamente distribuido en Sudamérica, Mesoamérica y México (Barneby, 1991; Morales \& Fortunato, 2010). La subserie Mimosa posee ocho especies actualmente reconocidas, seis de las cuales crecen en Sudamérica (Barneby, 1991; Morales \& Fortunato, 2010; Morales et al., 2012), con una gran diversidad morfológica y alta frecuencia de poliploides (Morales et al., 2010, 2012). En esta especie, los valores de los índices $\mathrm{A}_{1}, \mathrm{~A}_{2}$ y la alta proporción de cromosomas metacéntricos, son similares a los de otras especies diploides de Mimosa sección Mimosa, como M. xanthocentra Mart. y M. debilis Humb. \& Bonpl. ex Willd. (Morales et al., 2011). En estas especies se observa un índice $\mathrm{A}_{1}$ menor a 0,3 ; un índice $\mathrm{A}_{2}$ menor a 0,25 y en la fórmula cariotípica alrededor de las tres cuartas partes de los cromosomas son metacéntricos. El LCGH en las especies de la sección Mimosa generalmente es menor a $20 \mu \mathrm{m}$, tanto en diploides como en poliploides (Morales et al., 2014a). Esto muestra en general un cariotipo muy simétrico y con cromosomas pequeños en comparación con algunos representantes de la sección Batocaulon, en especial de la serie Farinosae.

Las diferencias estadísticamente significativas en LCGH y $\mathrm{A}_{1}$ permitirían distinguir a ambos taxones aquí estudiados, y en concordancia con los trabajos previos (Morales et al., 2011) diferenciar especies de la serie Farinosae y la subserie Mimosa. Hasta el momento, los estudios cariotípicos en especies de Mimosa sugieren que algunos parámetros serían de interés citotaxonómico en el género, aunque su relevancia debe confirmarse con estudios en un amplio rango de taxones.

\section{Agradecimientos}

Agradecemos al personal del Laboratorio de Citogenética, Ecología y Evolución (LACyE) de la Facultad de Ciencias Exactas y Naturales (Universidad de Buenos Aires) por su importante colaboración en el trabajo de rutina del laboratorio; asimismo, a los revisores del trabajo por sus valiosas sugerencias. Este trabajo recibió financiamiento a partir de los subsidios de la Universidad de Buenos Aires (UBA X178), CONICET (PIP 5560, 5927), Agencia de Promoción Científica y Tecnológica (PICT 0821-2011) y Universidad de Morón (PID B06-001/06, 06-001/08 y 06005/12).

\section{Bibliografía}

BARNEBY, R. C. 1991. Sensitivae Censitae: A description of the genus Mimosa Linnaeus (Mimosaceae) in the New World. Mem. New York Bot. Gard. 65: 1-835.

DAHMER N., M. F. SIMON, M. T. SCHIFINOWITTMANN, C. E. HUGHES, S. T. S. MIOTTO \& J. C. GIULIANI. 2011. Chromosome numbers in the genus Mimosa L.: cytotaxonomic and evolutionary implications. Plant Syst. Evol. 291: 211-220.

DI RIENZO, J. A., F. CASANOVES, M. G. BALZARINI, L. GONZÁLEZ, M. TABLADA \& C. W. ROBLEDO. 2009. InfoStat versión 2009. Grupo InfoStat, FCA, Universidad Nacional de Córdoba, Argentina.

GOLDBLATT, P. 1981. Cytology and the phylogeny of Leguminosae. En POLHILL, R. M. \& P. H. RAVEN (eds.), Advances of Legume Systematics 2, pp. 427-464. Royal Botanic Gardens, Kew.

ISELY, D. 1971. Legumes of the United States. IV. Mimosa. Am. Midl. Nat. 85: 410-424.

LEVAN, A., K. FREDGA \& A. A. SANDBERG. 1964. Nomenclature for centromeric position on chromosomes. Hereditas 52: 201-219.

MARÇAL DE SOUZA, S., A. CAMPOS REIS \& L. FACIO VICCINI. 2012. Polyploidy, B chromosomes, and heterochromatin characterization of Mimosa caesalpiniifolia Benth. (Fabaceae-Mimosoideae). Tree Genet. Genomes 9: 613-619.

MORALES, M. \& R. H. FORTUNATO. 2010. Novedades taxonómicas y nomenclaturales en el género Mimosa (Leguminosae) para el S de Sudamérica. Candollea 65: 169-184.

MORALES, M., A. F. WULFF, R. H. FORTUNATO \& L. POGGIO. 2010. Chromosome and morphological studies in the Mimosa debilis complex (Mimosoideae, Fabaceae) from Southern 
South America. Austr. J. Bot. 58: 12-22.

MORALES, M, A. F. WULFF, R. H. FORTUNATO \& L. POGGIO. 2011. Karyotype studies in Mimosa (Mimosoideae, Leguminosae) from Southern South America and ecological and taxonomic relationships. Caryologia 64: 203-214.

MORALES, M., O. S. RIBAS \& J. SANTOS-SILVA. 2012. A new polyploid species of Mimosa (Leguminosae, Mimosoideae) from the Highlands of Southern Brazil. Syst. Bot. 37: 399-403.

MORALES, M., A. F. WULFF, R. H. FORTUNATO \& L. POGGIO. 2014a. Chromosome studies in southern species of Mimosa (Fabaceae, Mimosoideae) and their taxonomic and evolutionary inferences. Pl. Syst. Evol. 300: 803-817.

MORALES, M., L. ARENAS, M. I. REMIS, A. F. WULFF, L. POGGIO \& R. H. FORTUNATO. 2014b. Morphometric and cytogenetic studies in Mimosa diversipila (Mimosoideae, Leguminosae) and their taxonomic and evolutionary inferences. Syst. Bot. 39: 875-883.

OLKOSKI, D. \& M. T. SCHIFINO WITTMANN. 2011. Cytogenetics of Mimosa bimucronata (DC.) O.
Kuntze (Mimosoideae, Leguminosae): chromosome number, polysomaty and meiosis. Crop Breed. Appl. Biotechnol. 11: 27-35.

REEVES, A. 2001. MicroMeasure: A new computer program for the collection and analysis of cytogenetic data. Genome 44: 359-443.

ROMERO-ZARCO, C. 1986. Anew method for estimating karyotype asymmetry. Taxon 35: 526-530.

SEIJO, G. J. 1993. Citogenética en especies argentinas del género Mimosa (Leguminosae). Bol. Soc. Argent. Bot. 29: 219-223.

SEIJO, G. J. 1999. Chromosome studies in Argentinian species of Mimosa. Cytologia 64: 241-246.

SEIJO, G. J. 2000. Números cromosómicos en especies de Mimosa de Paraguay. Bonplandia 10: 163-167.

SEIJO, G. J. \& A. FERNÁNDEZ. 2001. Chromosome numbers of some southernmost species of Mimosa $\mathrm{L}$. (Leguminosae). Cytologia 66: 19-34.

SIMON, M. F., R. GRETHER, L. P. QUEIROZ, T. E. SÄRKINEN, V. F. DUTRA \& C. E. HUGHES. 2011. The evolutionary history of Mimosa (Leguminosae): toward a phylogeny of the sensitive plants. Am. J. Bot. 98: 1201-1221.

Original recibido el 5 de marzo de 2015; aceptado el 30 de abril de 2015. 